\title{
On the Form of Smooth-Front Travelling Waves in a Reaction-Diffusion Equation with Degenerate Nonlinear Diffusion
}

\author{
J.A. Sherratt* \\ Department of Mathematics and Maxwell Institute for Mathematical Sciences, \\ Heriot-Watt University, Edinburgh EH14 4AS, UK
}

\begin{abstract}
Reaction-diffusion equations with degenerate nonlinear diffusion are in widespread use as models of biological phenomena. This paper begins with a survey of applications to ecology, cell biology and bacterial colony patterns. The author then reviews mathematical results on the existence of travelling wave front solutions of these equations, and their generation from given initial data. A detailed study is then presented of the form of smooth-front waves with speeds close to that of the (unique) sharp-front solution, for the particular equation $u_{t}=\left(u u_{x}\right)_{x}+u(1-u)$. Using singular perturbation theory, the author derives an asymptotic approximation to the wave, which gives valuable information about the structure of smooth-front solutions. The approximation compares well with numerical results.
\end{abstract}

Key words: nonlinear diffusion, travelling waves, sharp front, smooth front AMS subject classification: $35 \mathrm{~K} 57,35 \mathrm{~K} 65,92 \mathrm{~B} 05$

\section{Nonlinear Diffusion in Biological Models}

The first applications of reaction-diffusion equations in biology $[8,48]$ used standard linear diffusion to model population spread. However, it is now clear that in a number of biological contexts, motility varies with population density, requiring nonlinear diffusion terms. This was first realised in ecological applications $[12,13,45]$, and density-dependent dispersal is now a common feature

*E-mail: jas@ma.hw.ac.uk 
of spatial modelling in ecology. This includes models that are spatially discrete [33, 53] and of integrodifference equation type [51, 23], as well as reaction-diffusion equations (e.g. [9, 31, 52]). Empirically, positive and negative density dependence are both well-documented in a range of animal taxa $[28,22]$; see [37, 49] for modelling studies comparing the two cases.

Reaction-diffusion models with nonlinear diffusion have also been applied extensively to eukaryotic cell biology. In particular, [40] performed a detailed comparison of linear and nonlinear diffusion in a model of an in vitro experimental assay of cell migration and proliferation; they compared simulated wave front profiles with experimental data, concluding that the diffusion term giving the best fit with data depended on the cell type. A closely related study by [24] showed that for an in vitro wound healing assay, nonlinear diffusion was suggested by the shape of experimental cell density profiles, but was not required for a good fit with data. Nonlinear diffusion has also been used by a number of authors in models of interacting cell populations [41, 32, 47, 46], and has been applied specifically to wound repair $[42,5,50]$ and avascular tumour growth $[10,44,2,54]$.

A third application area for reaction-diffusion models with nonlinear diffusion is pattern formation in bacterial colonies. For a number of bacteria species exhibiting pattern formation in in vitro colonies, experimental observation of cell movement shows that it is an increasing function of both cell density and nutrient concentration. A number of different reaction-diffusion models have been proposed that incorporate this nonlinearity in cell motility (for example [20, 6, 27]).

In all three of these application areas, the key mathematical solution type is wave fronts. In ecology, such fronts correspond to invasions, a phenomenon of key ecological importance [14], which is occurring with increasing frequency as a result of climate change [15] in cell biology, wave fronts correspond to the advancing edge of an expanding cell population, such as a growing tumour; and in bacterial colonies, the tips extend as a wave front that is effectively one-dimensional [20]. Wave front behaviour is particularly interesting when the nonlinearity in diffusion is "degenerate", meaning that as the population density decreases towards zero, the diffusion coefficient does also. This case is relevant to all three application areas, and raises the possibility of sharp-front waves, as well as the smooth-front waves that arise in equations with linear diffusion (Figure 1). The distinction between these front types is that in sharp-front waves, the population density decreases to zero at a finite point in space, rather than decaying to zero asymptotically; sharp-front waves are sometimes known as "finite waves". This paper concerns these wave fronts in a particular reaction-diffusion equation with degenerate nonlinear diffusion.

In $\S 2$ I briefly review the literature on wave fronts in scalar reaction-diffusion equations with degenerate diffusion; there have been only a very few studies in corresponding systems of equations, all in the context of applications to bacterial colonies (e.g. [39, 7]). In $\S 3$ I discuss the evolution of travelling waves from given initial data, highlighting that numerical simulations suggest that the selection of a sharp- or smooth-front wave depends on the rate of decay of the initial data towards zero. $\S 4$ contains the major result of the paper, which involves using singular perturbation theory to study the form of smooth-front waves moving with speeds very close to that of the sharp-front wave. Finally, in $\S 5$, I discuss some extensions that could form the basis of future work. 


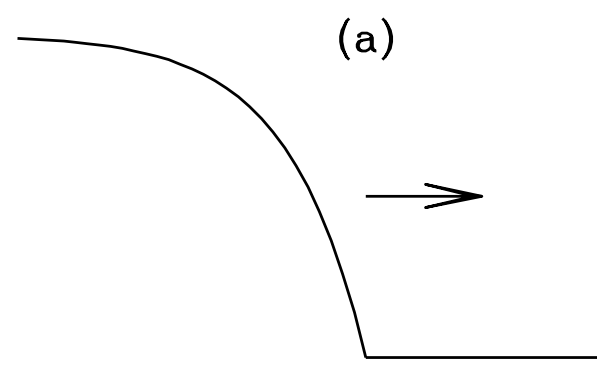

Sharp-front wave (b)

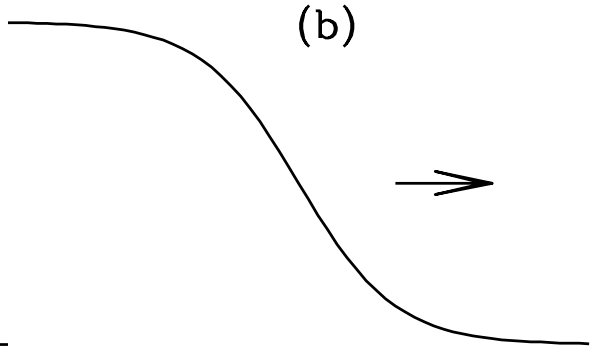

Smooth-front wave

Figure 1: A schematic illustration of the qualitative form of (a) a sharp-front travelling wave, and (b) a smooth-front travelling wave.

\section{Travelling Wave Fronts for Equations with Degenerate Dif- fusion}

Wave front solutions of reaction-diffusion equations with degenerate nonlinear diffusion were first studied thirty years ago [1, 30]. More recent work was initiated by Sánchez-Garduño \& Maini [34], who studied the equation

$$
\frac{\partial u}{\partial t}=\frac{\partial}{\partial x}\left[D(u) \frac{\partial u}{\partial x}\right]+f(u)
$$

with $D($.$) satisfying D(0)=0$ and $D^{\prime}(u)>0, D^{\prime \prime}(u) \neq 0$ on $[0,1]$, and $f($.$) satisfying f(0)=$ $f(1)=0, f^{\prime}(1)<0<f^{\prime}(0)$. They showed that travelling wave front solutions exist if and only if the wave speed $c \geq c^{*}$, with the wave being of sharp-front type if $c=c^{*}$ and smooth-front type otherwise. Travelling wave solutions have the form $u(x, t)=U(z)$ with $z=x-c t$, and therefore satisfy

$$
\begin{aligned}
d U / d z & =V \\
D(U) d V / d z & =-c V-D^{\prime}(U) V^{2}-f(U) .
\end{aligned}
$$

The singularity at $U=V=0$ can be removed via the change of variable

$$
\zeta=\int_{s=0}^{z} \frac{d s}{D(u(s))}
$$

$[1,36]$. In the case of a smooth-front wave, this transformation maps the real line onto itself, whereas for a sharp-front wave in which $U$ becomes zero at $z=z_{c},\left(-\infty, z_{c}\right)$ is mapped to $\mathbb{R}$. The resulting equations are

$$
\begin{aligned}
d U / d \zeta & =D(U) V \\
d V / d \zeta & =-c V-D^{\prime}(U) V^{2}-f(U) .
\end{aligned}
$$


which has three steady states: $(0,0),(0,-c)$ and $(1,0)$. Straightforward linear analysis shows that $(1,0)$ is a saddle point, so that there is exactly one trajectory, $\mathcal{T}$ say, leaving it and entering the fourth quadrant. Sánchez-Garduño \& Maini [34] showed that for some $c^{*}, \mathcal{T}$ terminates $(0,-\infty)$ if $c<c^{*}$, so that there is no travelling wave solution, while if $c>c^{*}, \mathcal{T}$ terminates at $(0,0)$, corresponding to a smooth-front travelling wave. If $c=c^{*}$, then $\mathcal{T}$ terminates at $\left(0,-c^{*}\right)$; thus at $\zeta=\infty$, corresponding to $z=z_{c}, U=0$ and $V=d U / d z$ is non-zero: the solution is a sharpfront wave. Phase portraits of (2.2) for these three cases are shown in Figure 2 for $D(u)=u$ and $f(u)=u(1-u)$.

The results of [34] have been extended to wider classes of functions $D($.$) and f($.$) by [36, 29,$ 26]. The first two of these papers use the same basic approach of analysing the phase portrait of (2.2), while the third uses a different method based on comparison techniques. Another approach to the proof, using shooting and the Conley index, is presented in [38]. Further extension to reactiondiffusion-convection equations has been considered in [11,25]. These authors study (2.1) with an additional term $h(u) \partial u / \partial x$ on the left hand side. Again there are wave front solutions if and only if the wave speed $c \geq c^{*}$, and again the wave is of smooth-front type for $c>c^{*}$. However in this case the minimum speed wave $\left(c=c^{*}\right)$ can be of smooth- or sharp-front type, according to whether $c^{*}>h(0)$ or $c^{*}=h(0)$ respectively. Malaguti \& Marcelli [25] give sufficient conditions for these two possibilities (and show that $c^{*} \geq h(0)$ in all cases).

In contrast to this extensive study of the existence of travelling wave solutions, their generation as the large-time limit as solutions of (2.1) with particular initial conditions has received relatively little attention. Sherratt \& Marchant [43] studied this question numerically for the case $D(u)=u$, $f(u)=u(1-u)$, for which $c^{*}=1 / \sqrt{2}$ (see below). They considered initial conditions

$$
u(x, 0)= \begin{cases}1 & \text { if } x<0 \\ e^{-\xi x} & \text { if } x>0\end{cases}
$$

$(0<\xi \leq \infty)$, and found that (2.3) always evolved towards a travelling wave solution, whose speed $c$ depended on $\xi$ via

$$
c=\left\{\begin{array}{lll}
1 / \sqrt{2} & \text { if } \xi \geq \sqrt{2} & \text { (sharp-front wave) } \\
1 / \xi & \text { if } \xi<\sqrt{2} & \text { (smooth-front wave) }
\end{array}\right.
$$

This dependence of wave speed on initial conditions can by explained intuitively via the observation that in (2.2) with $D(u)=u$ and $f(u)=u(1-u)$, the travelling wave trajectory approaches $(0,0)$ along the eigenvector $(-c, 1)$. (This eigenvector corresponds to an eigenvalue of zero, but has a stable centre manifold). Subsequently Biró [4], Medvedev [29] and Hilhorst [16] have proved that for particular classes of functions $D($.$) and f($.$) , solutions of (2.1) with compactly supported$ initial data evolve towards the travelling wave solution with minimum speed (i.e. the sharp-front wave). These results were extended by Kamin \& Rosenau $[18,19]$ to initial data that decays at a sufficiently fast rate at infinity. I am not aware of any rigorous work on convergence to smooth front waves for reaction-diffusion equations with degenerate nonlinear diffusion. 

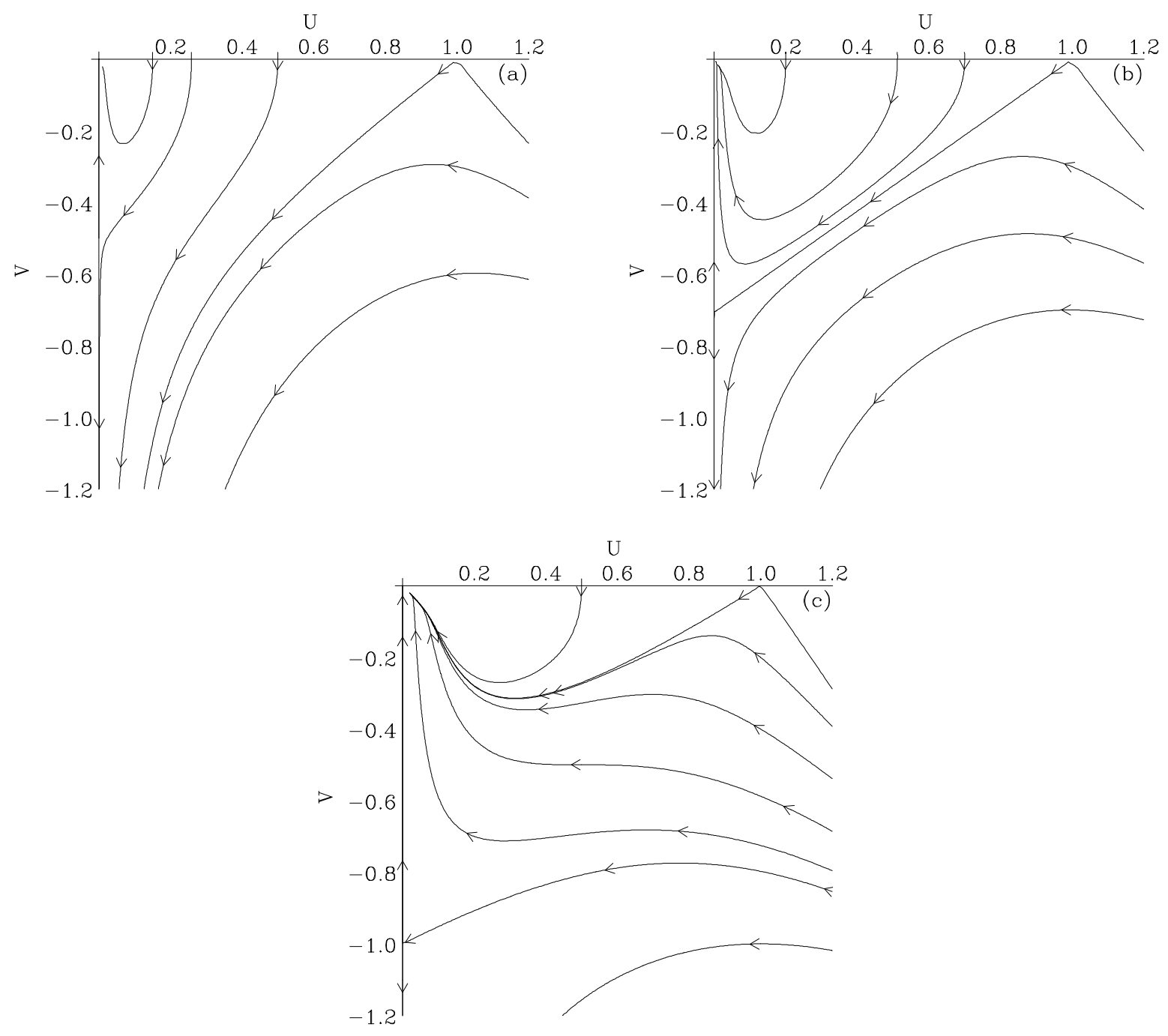

Figure 2: An illustration of the three basic qualitative forms of the phase portrait for the travelling wave ODEs (2.2). The phase portraits shown are for $D(u)=u$ and $f(u)=u(1-u)$ with (a) $c=0.5<c^{*}$; (b) $c=1 / \sqrt{2}=c^{*}$; (c) $c=1>c^{*}$. The qualitative form is the same for all $c \in(0,1 / \sqrt{2})$ and for all $c>1 / \sqrt{2}$. The ODEs were solved numerically using a Runge-KuttaMerson method. 


\section{The Form of Smooth-Front Waves}

In general, the minimum wave speed $c^{*}$ of travelling wave solutions of (2.1) is unknown (although bounds have been derived: see [3,26]. However for the special case $D(u)=u, f(u)=u(1-u)$, Aronson [1] showed that $c^{*}=1 / \sqrt{2}$, and moreover that the sharp-front wave is given by

$$
u^{*}(z)= \begin{cases}1-\exp \left(\frac{z-z_{c}}{\sqrt{2}}\right) & \text { if } z<z_{c} \\ 0 & \text { if } z>z_{c}\end{cases}
$$

This exact solution was exploited previously by Sánchez-Garduño \& Maini [35], who used regular perturbation theory to calculate the form of the sharp-front wave when small changes are made to the nonlinear diffusion coefficient $D($.$) . Here I fix D($.$) and f($.$) , and consider the form of the$ travelling wave solution when the wave speed $c$ is close to $c^{*}=1 / \sqrt{2}$. Specifically, I will derive an asymptotic expansion for the smooth-front wave of speed $\epsilon+1 / \sqrt{2}(\epsilon>0)$. The calculation is a standard application of singular perturbation theory: for general presentations, see for example the books by Hinch [17] and Kevorkian \& Cole [21].

The wave is a solution of the travelling wave equation

$$
U \frac{d^{2} U}{d z^{2}}+\left(\frac{1}{\sqrt{2}}+\epsilon\right) \frac{d U}{d z}+\left(\frac{d U}{d z}\right)^{2}+U(1-U)=0
$$

subject to the boundary conditions $U(-\infty)=1$ and $U(+\infty)=0$. This boundary value problem does of course have a solution when $\epsilon=0$, namely the sharp-front travelling wave (3.1). Nevertheless, this is not a regular perturbation problem since the $\epsilon=0$ solution (3.1) is non-smooth at $z=z_{c}$. Therefore one expects the solution to have the form of two outer expansions on either side of $z=z_{c}$, with a corner layer centred on $z=z_{c}$ to give a smooth transition between these outer solutions. Without loss of generality, and to simplify the algebra, I take $z_{c}=0$.

\section{Inner and Outer Solutions}

In the region $z<z_{c}=0$, I look initially for a solution of (3.2) with a simple power series form:

$$
U_{\text {left }}(z ; \epsilon)=U_{\text {left }, 0}(z)+\epsilon U_{\text {left }, 1}(z)+\epsilon^{2} U_{\text {left }, 2}(z)+\ldots .
$$

Here the subscript $t_{\text {left }}$ indicates that this solution applies in the outer region to the left of the corner layer. I already know that $U_{\text {left, } 0}(z)=1-\exp (z / \sqrt{2})$, and substituting this together with the expansion (3.3) into the governing equation (3.2), and equating powers of $\epsilon$, gives a linear equation for $U_{\text {left }, 1}(z)$ :

$$
\frac{d^{2} U_{\text {left }, 1}}{d z^{2}}\left(1-e^{z / \sqrt{2}}\right)+\frac{d U_{\text {left }, 1}}{d z}\left(\frac{1}{\sqrt{2}}-\sqrt{2} e^{z / \sqrt{2}}\right)+U_{\text {left }, 1}\left(\frac{3}{2} e^{z / \sqrt{2}}-1\right)=\frac{e^{z / \sqrt{2}}}{\sqrt{2}} .
$$

One solution of the homogeneous equation corresponding to (3.4) can be found immediately by inspection: $U_{\text {left, } 1}(z)=\exp (z / \sqrt{2})$. The other homogeneous solution can thus be found in a 
standard way by substituting $U_{\text {left, } 1}(z)=\widehat{U}_{\text {left, } 1}(z) \exp (z / \sqrt{2})$, and a particular integral can then be constructed using the method of variation of parameters. Applying the boundary condition $U_{\text {left }, 1}(-\infty)=0$ removes one constant of integration, giving the following solution:

$$
U_{\text {left }, 1}=A_{\text {left }, 1} e^{z / \sqrt{2}}+\frac{e^{z / \sqrt{2}}}{9 \sqrt{2}}\left[3 \sqrt{2} z-6 \log \left(1-e^{z / \sqrt{2}}\right)-2\right] .
$$

Here $A_{\text {left, } 1}$ is the outstanding constant of integration.

The procedure for obtaining the outer solution in the region $z>0$ is very similar. I look for a solution of the form $U_{\text {right }}(z ; \epsilon)=U_{\text {right }, 0}+\epsilon U_{\text {right, } 1}+\epsilon^{2} U_{\text {right, } 2}+\ldots$ In this case, $U_{\text {right }, 0}=0$, and substituting into (3.2) gives a very simple equation for $U_{\text {right, } 1}$ :

$$
d U_{\text {right }, 1} / d z=-\sqrt{2} U_{\text {right }, 1} \quad \Longrightarrow \quad U_{\text {right }, 1}(z)=A_{\text {right }, 1} \exp (-\sqrt{2} z)
$$

where $A_{\text {right, } 1}$ is a constant of integration.

I consider now the corner layer centred on $z=0$. The rescaled variables in this layer will have the form $\widetilde{U}=U / \mu(\epsilon), \widetilde{z}=z / \nu(\epsilon)$, where $\mu(\epsilon)=\mathcal{O}(1)$ and $\nu(\epsilon)=o(1)$ as $\epsilon \rightarrow 0$. Substituting these rescalings into (3.2) shows clearly that the appropriate scaling has $\mu(\epsilon) \sim \nu(\epsilon)$, in which case the equation becomes

$$
\widetilde{U} \frac{d^{2} \widetilde{U}}{d \widetilde{z}^{2}}+\frac{1}{\sqrt{2}} \frac{d \widetilde{U}}{d \widetilde{z}}+\left(\frac{d \widetilde{U}}{d \widetilde{z}}\right)^{2}+\epsilon \frac{d \widetilde{U}}{d \widetilde{z}}+\nu(\epsilon) \widetilde{U}-\nu(\epsilon)^{2} \widetilde{U^{2}}=0 .
$$

Again I look for a power series expansion

$$
\widetilde{U}(\widetilde{z} ; \epsilon)=\widetilde{U}_{0}(\widetilde{z})+\epsilon \widetilde{U}_{1}(\widetilde{z})+\epsilon^{2} \widetilde{U}_{2}(\widetilde{z})+\ldots .
$$

Substituting into (3.7) gives an equation for $\widetilde{U}_{0}$ which can easily be solved; the solution has the implicit form

$$
\widetilde{A}_{0}-\widetilde{z} / \sqrt{2}=\widetilde{U}_{0}+\widetilde{B}_{0} \log \left|\widetilde{U}_{0}-\widetilde{B}_{0}\right| .
$$

Here $\widetilde{A}_{0}$ and $\widetilde{B}_{0}$ are constants of integration. I am looking for a solution in which $\widetilde{z}$ runs continuously from $-\infty$ to $+\infty$, with $\widetilde{U}_{0}$ always positive. Simple sketching of the $\widetilde{z}-\widetilde{U}_{0}$ graph implied by (3.9) shows that $\widetilde{B}_{0}$ must be positive, and that $\widetilde{U}_{0}$ decreases monotonically from $+\infty$ to $\widetilde{B}_{0}$ as $\widetilde{z}$ increases from $-\infty$ to $\infty$; the form of $\widetilde{U}_{0}$ is illustrated in Figure 3.

\section{Matching and Intermediate Terms}

I expect the four outstanding constants of integration to be determined by matching the outer and corner layer solutions. In order to consider this matching, I must obtain the forms of $\widetilde{U}_{0}$ as $\widetilde{z} \rightarrow$ $\pm \infty$ in more detail. Consider first the $\widetilde{z} \rightarrow+\infty$ limit, for which $\widetilde{U}_{0} \sim \widetilde{B}_{0}$. I let $\gamma(\widetilde{z})=\widetilde{U}_{0}-\widetilde{B}_{0}$. Substituting this into the implicit equation (3.9) which defines $\widetilde{U}_{0}$ gives $\gamma+\widetilde{B}_{0} \log \gamma=\widetilde{A}_{0}-\widetilde{z} / \sqrt{2}$. Thus

$$
\widetilde{U}_{0}(\widetilde{z})=\widetilde{B}_{0}+\mathcal{O}_{s}\left[\exp \left(-\widetilde{z} / \widetilde{B}_{0} \sqrt{2}\right)\right] \text { as } \widetilde{z} \rightarrow+\infty .
$$




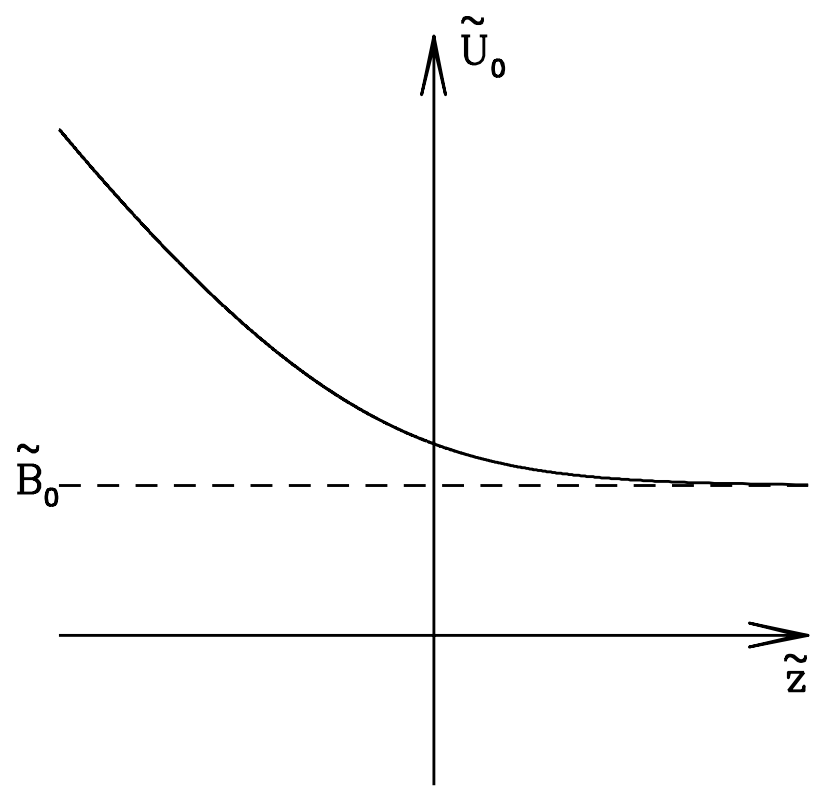

Figure 3: An illustration of the qualitative form of $\widetilde{U}_{0}(\widetilde{z})$, defined by the implicit formula (3.9).

A similar procedure of successive approximation shows that

$$
\widetilde{U}_{0}(\widetilde{z})=-\widetilde{z} / \sqrt{2}-\widetilde{B}_{0} \log (-\widetilde{z})+\left(\widetilde{A}_{0}+\widetilde{B}_{0} \log \sqrt{2}\right)+\mathcal{O}[\log (-\widetilde{z}) / \widetilde{z}] \text { as } \widetilde{z} \rightarrow-\infty .
$$

I am now in a position to consider matching the outer and layer solutions. For this purpose I introduce an intermediate variable $z_{\eta}=z / \eta(\epsilon)=\widetilde{z} \cdot \nu(\epsilon) / \eta(\epsilon)$ with $\nu(\epsilon) \ll \eta(\epsilon) \ll 1$. For notational simplicity, I will simply write $\nu$ and $\eta$, without the " $(\epsilon)$ ", henceforth. The conditions for matching to some order $M(\epsilon)$ are then

$$
\begin{aligned}
{\left[U_{\text {right }}\left(\eta z_{\eta} ; \epsilon\right)-\nu \widetilde{U}\left(\eta z_{\eta} / \nu\right)\right] } & =o[M(\epsilon)] \text { as } \quad \epsilon \rightarrow 0 \text { with } z_{\eta}>0 \text { fixed } \\
\text { and }\left[U_{\text {left }}\left(\eta z_{\eta} ; \epsilon\right)-\nu \widetilde{U}\left(\eta z_{\eta} / \nu\right)\right] & =o[M(\epsilon)] \text { as } \quad \epsilon \rightarrow 0 \text { with } z_{\eta}<0 \text { fixed. }
\end{aligned}
$$

Explicitly, the first of these conditions is

$$
\begin{gathered}
\overbrace{0}^{U_{\text {right, } 0}} \overbrace{+\epsilon A_{\text {right }, 1}-A_{\text {right, } 1} \sqrt{2} \epsilon \eta z_{\eta}+\mathcal{O}\left(\epsilon \eta^{2}\right)}^{\epsilon U_{\text {right, } 1}}+\overbrace{o(\epsilon)}^{\epsilon} \overbrace{\nu}^{U_{\text {right, } 2}}+\ldots \\
= \\
\underbrace{\nu \widetilde{B}_{0}+\mathcal{O}\left(\nu \exp \left[-\eta z_{\eta} /\left(\nu \widetilde{B}_{0} \sqrt{2}\right)\right]\right)}_{\nu \widetilde{U}_{0}} \underbrace{-\widetilde{B}_{0} \sqrt{2} \epsilon \eta z_{\eta}+o(\epsilon \eta)}_{\nu \epsilon \widetilde{U}_{1}+\ldots}+o[M(\epsilon)]
\end{gathered}
$$

as $\epsilon \rightarrow 0$ with $z_{\eta}>0$ fixed. Here I have used (3.10), and I have expanded the outer solutions (3.6) in Taylor series about $z=0$. I have also included the leading order contributions of the $\mathcal{O}(\epsilon)$ 
terms in the corner layer series (3.8), to emphasise that these contributions do match other terms in the outer expansion. These terms do of course require calculation. The equation determining $\widetilde{U}_{1}$ is most conveniently expressed as

$$
\widetilde{U}_{0} \frac{d^{2} \widetilde{U}_{1}}{d \widetilde{z}^{2}}+\left(\frac{\widetilde{B}_{0} \sqrt{2}}{\widetilde{U}_{0}}-\frac{1}{\sqrt{2}}\right) \frac{d \widetilde{U}_{1}}{d \widetilde{z}}+\frac{\widetilde{B}_{0}\left(\widetilde{U}_{0}-\widetilde{B}_{0}\right)}{2 \widetilde{U}_{0}^{3}} \widetilde{U}_{1}=\frac{1}{\sqrt{2}}-\widetilde{U}_{0}-\frac{\widetilde{B}_{0}}{\widetilde{U}_{0} \sqrt{2}} .
$$

Since I have already calculated the behaviour of $\widetilde{U}_{0}$ as $\widetilde{z} \rightarrow \pm \infty$, (3.13) can be used, without determining a full solution, to find the corresponding behaviour of $\widetilde{U}_{1}$. For this I use a method of successive approximation similar to that used in deriving (3.10) and (3.11). This rather crude method works because any particular integral of (3.13) dominates the solution of the corresponding homogeneous equation both as $z \rightarrow+\infty$ and as $z \rightarrow-\infty$.

Matching to order unity, so that $M(\epsilon) \sim 1$, is automatic, and higher order matching clearly requires $\nu(\epsilon)=\epsilon$. In this case, (3.12) with $M(\epsilon)=\epsilon$ implies that $A_{\text {right, } 1}=\widetilde{B}_{0}$. However, in the corresponding matching condition to the left of the corner layer, matching to order $\epsilon$ is not possible, because there is an unbalanced term of order $\nu \log \nu=\epsilon \log \epsilon$ in the layer solution. To deal with this I introduce the additional intermediate term $\epsilon \log \epsilon U_{\text {left, } \log }(z)$ into the expansion (3.3) for $U_{\text {left }}$. Such intermediate terms are a common feature of matched asymptotic expansions: see $\S 5.2$ of [17] or $\S 2.3-2.4$ of [21] for general discussions. A separate equation must be solved

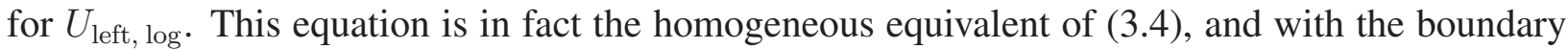
condition $U_{\text {left, } \log }(-\infty)=0$, the solution is $U_{\text {left, } \log }(z)=A_{\text {left, } \log } \exp (z / \sqrt{2})$, where $A_{\text {left, } \log }$ is a constant of integration. Expanding this in a Taylor series about $z=0$ gives the following amended matching condition to the left of the layer

$$
\begin{aligned}
& \overbrace{-\eta z_{\eta} / \sqrt{2}-\eta^{2} z_{\eta}^{2} / 4+\mathcal{O}\left(\eta^{3}\right)}^{U_{\text {left, } 0}} \overbrace{+A_{\text {left, } \log } \epsilon \log \epsilon+\left(A_{\text {left, } \log } / \sqrt{2}\right) \eta z_{\eta} \epsilon \log \epsilon+\mathcal{O}\left(\eta^{2} \epsilon \log \epsilon\right)}^{\epsilon \log \epsilon U_{\text {left, } \log }} \\
& \epsilon U_{\text {left, } 1} \text { (continued on next line) } \\
& \overbrace{+\epsilon A_{\text {left }, 1}+\epsilon \eta A_{\text {left }, 1} z_{\eta} / \sqrt{2}+\mathcal{O}\left(\epsilon \eta^{2}\right)-\epsilon \sqrt{2} / 9+\epsilon \eta z_{\eta} / 18-(\sqrt{2} / 3) \epsilon \log \left(-z_{\eta} / \sqrt{2}\right)} \\
& \epsilon U_{\text {left, } 1} \text { (continued from previous line) } \\
& \overbrace{-(\sqrt{2} / 3) \epsilon \log \eta-(1 / 3) \epsilon \eta z_{\eta} \log \eta-(1 / 3) \epsilon \eta z_{\eta} \log \left(-z_{\eta} / \sqrt{2}\right)+\mathcal{O}\left(\epsilon \eta^{2}\right)}^{\epsilon^{2} U_{\text {left, } 2}+\ldots}+\overbrace{o(\epsilon)}^{+.}= \\
& \nu \widetilde{U}_{0} \\
& \overbrace{-\eta z_{\eta} / \sqrt{2}-\epsilon \widetilde{B}_{0} \log \eta+\epsilon \widetilde{B}_{0} \log \epsilon-\epsilon \widetilde{B}_{0} \log \left(-z_{\eta}\right)+\epsilon\left(\widetilde{A}_{0}+\widetilde{B}_{0} \log \sqrt{2}\right)+\mathcal{O}\left[\left(\epsilon^{2} / \eta\right) \log \eta\right]} \\
& \epsilon \nu \widetilde{U}_{1}+\ldots \\
& \overbrace{-\eta^{2} z_{\eta}^{2} / 4-\left(\widetilde{B}_{0} z_{\eta} / \sqrt{2}\right) \epsilon \eta \log \left(-\eta z_{\eta} / \epsilon\right)+\left[\widetilde{A}_{0}+\widetilde{B}_{0} \log \sqrt{2}+7 \widetilde{B}_{0} / 2-\sqrt{2}\right] \epsilon \eta z_{\eta} / \sqrt{2}+o(\epsilon \eta)} \\
& +o[M(\epsilon)]
\end{aligned}
$$


The conditions for matching to order $\epsilon$, so that $M(\epsilon) \sim \epsilon$, are thus $A_{\text {left, } \log }=\widetilde{B}_{0}, \widetilde{B}_{0}=\sqrt{2} / 3$ and $A_{\text {left }, 1}=\widetilde{A}_{0}+\sqrt{2} / 9$. This last relation is obtained by equating coefficients of $\epsilon$. The constant $\widetilde{A}_{0}$ is not determined, and corresponds to an $\mathcal{O}(\epsilon)$ translation in the wave coordinate $z$. (Recall that both the equation (3.2) and the boundary conditions are autonomous, although $z$ is fixed at $\mathcal{O}(1)$ by the specification of $z_{c}$ ).

\section{Composite Expansion and Conclusions}

Having matched the outer and corner layer solutions, I can calculate a leading order composite generalised asymptotic expansion in the usual way, by adding the two solutions and subtracting the common matched values. The defining property of this composite expansion $U_{\text {comp }}(z)$ is that the difference between $U_{\text {comp }}(z)$ and the outer solution is $o(\epsilon)$ as $\epsilon \rightarrow 0$ with $z$ fixed, while the difference between $U_{\text {comp }}(z)$ and the corner layer solution is $o(\epsilon)$ as $\epsilon \rightarrow 0$ with $\widetilde{z}=z / \epsilon$ fixed. The form of $U_{\text {comp }}$ is:

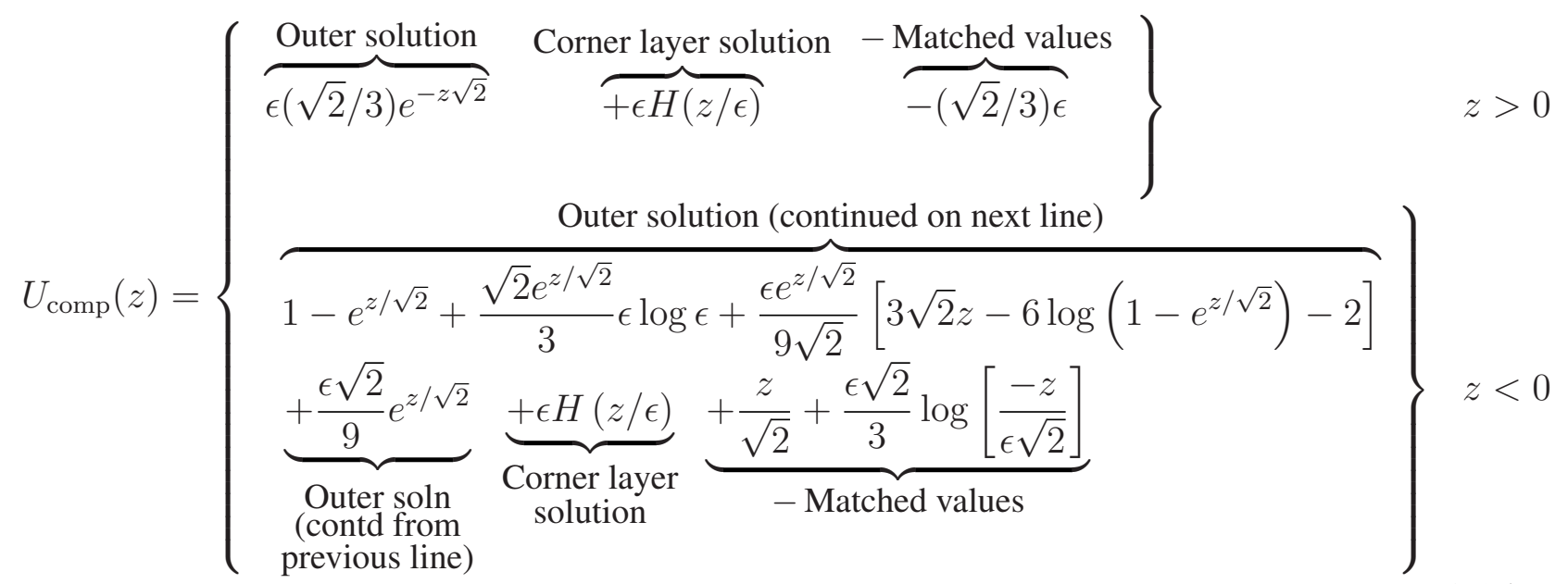

Here I have taken $\widetilde{A}_{0}=0$. The function $H(\xi)$ is the unique solution on $(\sqrt{2} / 3, \infty)$ of

$$
\sqrt{2} H(\xi)+(2 / 3) \log [H(\xi)-\sqrt{2} / 3]+\xi=0 .
$$

This composite expansion approximates well with the numerically calculated travelling wave profile provided $\epsilon$ is small (Figure 4a). However the relatively large leading order correction $(\mathcal{O}(\epsilon \log \epsilon))$ means that the quality of the approximation decreases relatively rapidly as $\epsilon$ increases (Figure $4 b$ ).

The leading order composite solution (3.15) is the net result of our matched asymptotics. Higher order corrections could of course be obtained in a similar way. One application of this solution is as an approximation to the true wave form, although the algebraic complexity of (3.15) means that this has little practical advantage over a numerical approximation, which can easily be found as explained above. A much more significant application of (3.15) is the insight it gives into the structure of the smooth-front travelling waves of speed close to the minimum speed. The 

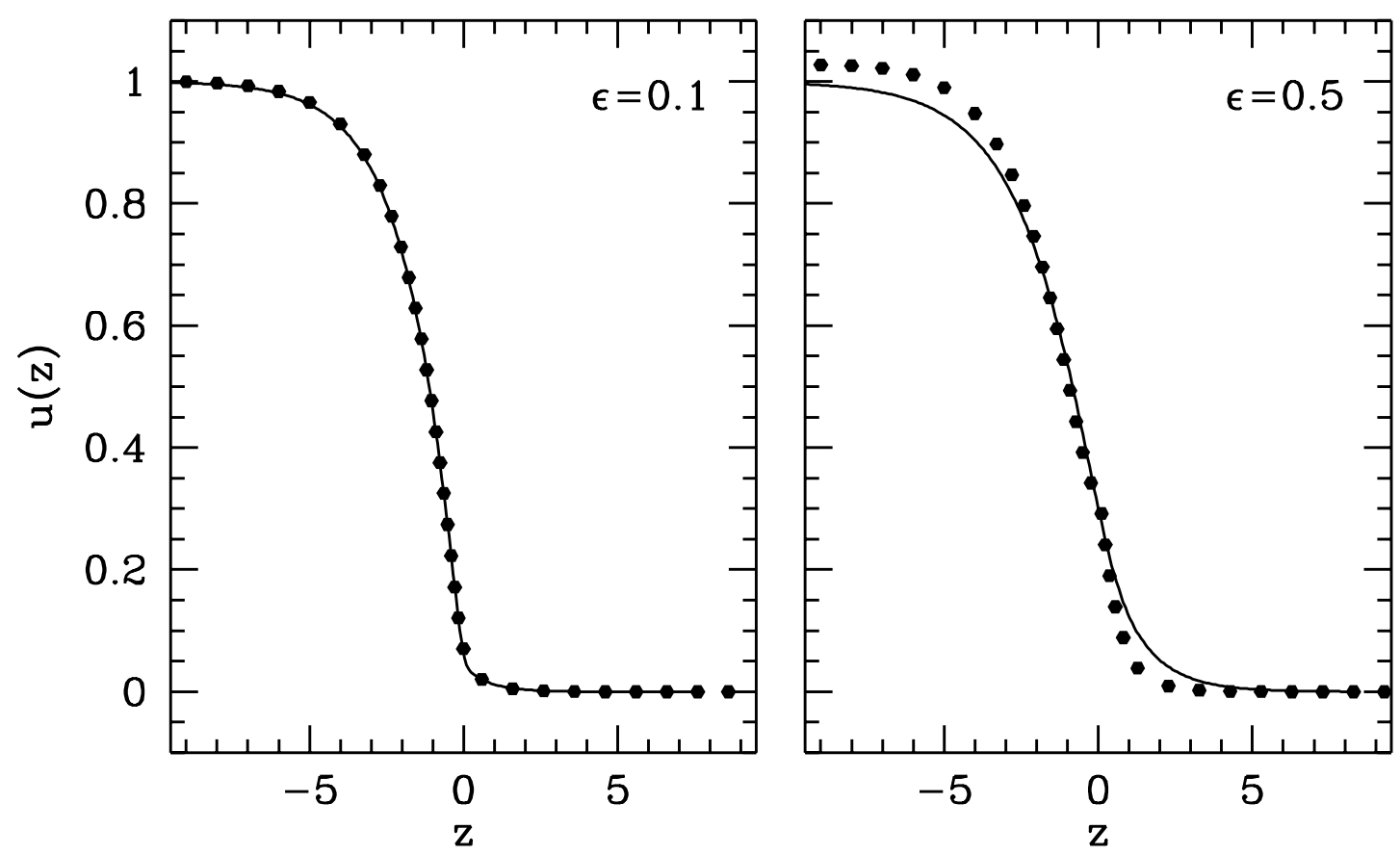

Figure 4: A comparison of the leading order composite expansion $U_{\text {comp }}(z)$ (points) with the numerically calculated travelling wave (solid curve), for $\epsilon=0.1$ and $\epsilon=0.5$. There is almost no visible difference in the former case, but when $\epsilon$ is as large as 0.5 there is a significant difference. Note that $\epsilon=0.5$ gives a wave speed that is about $70 \%$ greater than the minimum speed of $1 / \sqrt{2}$. The numerically calculated wave was determined by integrating the partial differential equation (2.1) with $D(u)=u$ and $f(u)=u(1-u)$ from initial conditions satisfying (2.3) with $\xi=1 /(\epsilon+1 / \sqrt{2})$. I solved on the domain $-23<x<50$ up to time $t=40 / c$, where $c$ is the expected wave speed, given by (2.4), so that $c=\epsilon+1 / \sqrt{2}$. This solution time is sufficiently long to allow the wave form to establish, but short enough that there is no significant interaction between the wave and the boundary at $x=50$. An appropriate translation in space was applied prior to plotting. The numerical method was the method of lines and Gear's method 
simple fact that there is a corner layer of width $\epsilon$ in which the solution is of order $\epsilon$ is the most noticeable aspect of this. But perhaps more significant is the requirement to introduce an intermediate term to the left of the layer. A simple implication of this is that the maximum difference between the smooth-front and sharp-front waves is $\mathcal{O}_{s}(\epsilon \log \epsilon)$ as $\epsilon \rightarrow 0$.

\section{Possible Extensions for Future Work}

To the best of my knowledge, the work presented here is the first detailed investigation of the form of smooth-front wave solutions of a reaction-diffusion equation with degenerate nonlinear diffusion, and it raises a number of challenges for future work. Firstly, my results apply only to the specific case $D(u)=u, f(u)=u(1-u)$. A natural extension would be to consider more general $D($.$) and f($.$) . This is a more difficult problem due to the lack of an exact leading order solution,$ but may be within reach given the detailed knowledge of the phase portraits of the travelling wave equations $(2.2)$ [34, 36, 29]. A key question is whether changes in $D($.$) and f($.$) can alter the$ dependence on $\epsilon$ of the leading order correction to the wave form, which is $\mathcal{O}(\epsilon \log \epsilon)$ for the case I have considered. A second natural extension would be to reaction-diffusion-convection equations. For various equations of this type, Gilding \& Kersner [11] give exact solutions for the minimum speed wave, which would enable studies directly parallelling that presented here. Again, a key question concerns the dependence on $\epsilon$ of the leading order correction to the wave form, and in particular how this varies with the convection coefficient. A third and much more challenging area for future work is the proof of rigorous results on the evolution of slowly decaying initial data to smooth-front waves.

\section{Acknowledgements}

This work was supported in part by a Leverhulme Trust Research Fellowship. I am very grateful to Ben Marchant for initiating my interest in this problem.

\section{References}

[1] D.G. Aronson. Density dependent interaction systems. In: W.H. Steward et al. (eds.) Dynamics and Modelling of Reactive Systems, pp. 1161-176. Academic Press, New York, 1980.

[2] B.P. Ayati, G.F. Webb, A.R.A. Anderson. Computational methods and results for structured multiscale models of tumor invasion. Multiscale Modeling \& Simulation, 5 (2006), 1-20.

[3] R.D. Benguria, M.C. Depassier. Speed of fronts of the reaction-diffusion equation. Phys. Rev. Lett., 77 (1996), 1171-1173. 
[4] Z. Biró. Stability of travelling waves for degenerate reaction-diffusion equations of KPP-type. Adv. Nonlinear Stud., 2 (2002), 357-371.

[5] A.Q. Cai, K.A. Landman, B.D. Hughes. Multi-scale modelling of a wound healing cell migration assay. J. Theor. Biol., 245 (2007), 576-594.

[6] I. Cohen, I. Golding, Y. Kozlovsky, E. Ben-Jacob, I.G. Ron. Continuous and discrete models of cooperation in complex bacterial colonies. Fractals, 7 (1999), 235-247.

[7] P. Feng, Z. Zhou. Finite traveling wave solutions in a degenerate cross-diffusion model for bacterial colony. Comm. Pure Appl. Anal., 6 (2007), 1145-1165.

[8] R.A. Fisher. The wave of advance of advantageous genes. Ann. Eugenics, 7 (1937), 353-369.

[9] G. García-Ramos, F. Sánchez-Garduño, P.K. Maini. Dispersal can sharpen parapatric boundaries on a spatially varying environment. Ecology, 81 (2000), 749-760.

[10] R.A. Gatenby, E.T. Gawlinski. A reaction-diffusion model of cancer invasion. Cancer Res., 56 (1996), 4740-4743.

[11] B.H. Gilding, R. Kersner. A Fisher/KPP-type equation with density-dependent diffusion and convection: travelling-wave solutions. J. Phys. A: Math. Gen., 38 (2005), 3367-3379.

[12] W.S.C. Gurney, R.M. Nisbet. The regulation of inhomogeneous population. J. Theor. Biol., 52 (1975), 441-457.

[13] W.S.C. Gurney, R.M. Nisbet. A note on nonlinear population transport. J. Theor. Biol., 56 (1976), 249-251.

[14] A. Hastings, K. Cuddington, K.F. Davies, C.J. Dugaw, S. Elmendorf, A. Freestone, S. Harrison, M. Holland, J. Lambrinos, U. Malvadkar, B.A. Melbourne, K. Moore, C. Taylor, D. Thomson. The spatial spread of invasions: new developments in theory and evidence. Ecol. Lett., 8 (2005), 91-101.

[15] J.J. Hellmann, J.E. Byers, B.G. Bierwagen, J.S. Dukes. Five potential consequences of climate change for invasive species. Conserv. Biol., 22 (2008), 534-543.

[16] D. Hilhorst, R. Kersner, E. Logak, M. Mimura. Interface dynamics of the Fisher equation with degenerate diffusion. J. Differential Equations, 244 (2008), 2870-2889.

[17] E.J. Hinch. Perturbation Methods. Cambridge University Press, 1991.

[18] S. Kamin, P. Rosenau. Convergence to the travelling wave solution for a nonlinear reactiondiffusion equation. Rend. Mat. Acc. Lincei, 15 (2004a), 271-280.

[19] S. Kamin, P. Rosenau. Emergence of waves in a nonlinear convection-reaction-diffusion equation. Adv. Nonlinear Stud., 4 (2004b), 251-272. 
[20] K. Kawasaki, A. Mochizuki, M. Matsushita, T. Umeda, N. Shigesada. Modeling spatiotemporal patterns generated by Bacillus subtilis. J. Theor. Biol., 188 (1997), 177-185.

[21] J. Kevorkian, J.D. Cole. Multiple Scale and Singular Perturbation Methods. Springer-Verlag, New York, 1996.

[22] S.Y. Kim, R. Torres, H. Drummond. Simultaneous positive and negative density-dependent dispersal in a colonial bird species. Ecology, 90 (2009), 230-239.

[23] F. Lutscher. Density-dependent dispersal in integrodifference equations. J. Math. Biol., 56 (2008), 499-524.

[24] P.K. Maini, S. McElwain, D. Leavesley. A travelling wave model to interpret a wound healing migration assay for human peritoneal mesothelial cells. Tissue Eng., 10 (2004), 475-482.

[25] L. Malaguti, C. Marcelli. Finite speed of propagation in monostable degenerate reactiondiffusion-convection equations. Advanced Nonlinear Studies, 5 (2005), 223-252.

[26] L. Malaguti, C. Marcelli. Sharp profiles in degenerate and doubly degenerate Fisher-KPP equations. J. Differential Equations, 195 (2003), 471-496.

[27] M.B.A. Mansour. Traveling wave solutions of a nonlinear reaction-diffusion-chemotaxis model for bacterial pattern formation. Applied Mathematical Modelling, 32 (2008), 240247.

[28] E. Matthysen. Density-dependent dispersal in birds and mammals. Ecography, 28 (2005), 403-416.

[29] G.S. Medvedev, K. Ono, P.J. Holmes. Travelling wave solutions of the degenerate Kolmogorov-Petrovski-Piskunov equation. Eur. J. Appl. Math., 14 (2003) 343-367.

[30] W.I. Newman. Some exact solutions to a nonlinear diffusion problem in population genetics and combustion. J. Theor. Biol., 85 (1980), 325-334.

[31] A. Okubo, A. Hastings, T. Powell. Population dynamics in temporal and spatial domains. In: A. Okubo, S.A. Levin (eds.) Diffusion and ecological problems: modern perspectives, pp. 298-373. Springer, New York, 2001.

[32] K.J. Painter, J.A. Sherratt. Modelling the movement of interacting cell populations. J. Theor. Biol., 225 (2003), 327-339.

[33] B.-E. Saether, S. Engen, R. Lande. Finite metapopulation models with density-dependent migration and stochastic local dynamics. Proc. R. Soc. Lond. B, 266 (1999), 113-118.

[34] F. Sánchez-Garduño, P.K. Maini. Existence and uniqueness of a sharp front travelling wave in degenerate nonlinear diffusion Fisher-KPP equations. J. Math. Biol., 33 (1994a), 163-192. 
[35] F. Sánchez-Garduño, P.K. Maini. An approximation to a sharp front type solution of a density dependent reaction-diffusion equation. Appl. Math. Lett., 7 (1994b), 47-51.

[36] F. Sánchez-Garduño, P.K. Maini. Travelling wave phenomena in some degenerate reactiondiffusion equations. J. Differential Equations, 117 (1995), 281-319.

[37] F. Sánchez-Garduño, P.K. Maini, E. Kappos. A review on travelling wave solutions of one-dimensional reaction diffusion equations with non-linear diffusion term. FORMA, 11 (1996a), 45-59.

[38] F. Sánchez-Garduño, E. Kappos, P.K. Maini. A shooting argument approach to a sharp type solution for nonlinear degenerate Fisher-KPP equations. IMA J. Appl. Math. 57 (1996b), 211-221.

[39] R.A. Satnoianu, P.K. Maini, F. Sánchez-Garduño, J.P. Armitage. Travelling waves in a nonlinear degenerate diffusion model for bacterial pattern formation. Discrete and Continuous Dynamical Systems B, 1 (2001), 339-362.

[40] B.G. Sengers, C.P. Please, R.O.C. Oreffo. Experimental characterization and computational modelling of two-dimensional cell spreading for skeletal regeneration. J. R. Soc. Interface, 4 (2007), 1107-1117.

[41] J.A. Sherratt. Wave front propagation in a competition equation with a new motility term modelling contact inhibition between cell populations. Proc. R. Soc. Lond. A 456 (2000), 2365-2386.

[42] J.A. Sherratt, J.D. Murray. Models of epidermal wound healing. Proc. R. Soc. Lond. B, 241 (1990), 29-36.

[43] J.A. Sherratt, B.P. Marchant. Non-sharp travelling wave fronts in the Fisher equation with degenerate nonlinear diffusion. Appl. Math. Lett. 9 (1996), 33-38.

[44] J.A. Sherratt, M.A.J. Chaplain. A new mathematical model for avascular tumour growth. J. Math. Biol., 43 (2001), 291-312.

[45] N. Shigesada, K. Kawasaki, E. Teramoto. Spatial segregation of interacting species. J. Math. Biol., 79 (1979), 83-99.

[46] M.J. Simpson, K.A. Landman, K. Bhaganagarapu. Coalescence of interacting cell populations. J. Theor. Biol. 247 (2007), 525-543.

[47] M.J. Simpson, K.A. Landman, B.D. Hughes, D.F. Newgreen. Looking inside an invasion wave of cells using continuum models: proliferation is the key. J. Theor. Biol., 243 (2006), 343-360.

[48] J.G. Skellam. Random dispersal in theoretical populations. Biometrika, 38 (1951), 196-218. 
[49] M.J. Smith, J.A. Sherratt, X. Lambin. The effects of density-dependent dispersal on the spatiotemporal dynamics of cyclic populations. J. Theor. Biol., 254 (2008), 264-274.

[50] A. Tremel, A. Cai, N. Tirtaatmadja, B.D. Hughes, G.W. Stevens, K.A. Landman, A.J. OConnor. Cell migration and proliferation during monolayer formation and wound healing. Chem. Eng. Sci., 64 (2009), 247-253.

[51] R.R. Veit, M.A. Lewis. Integrodifference models for persistence in fragmented habitats. Bull. Math. Biol., 59 (1997), 107-137.

[52] Y. Wu. Existence of stationary solutions with transition layers for a class of cross-diffusion systems. Proc. R. Soc. Ed., 132A (2002), 1493-1511.

[53] J. Ylikarjula, S. Alaja, J. Laakso, D. Tesar. Effects of patch number and dispersal patterns on population dynamics and synchrony. J. Theor. Biol., 207 (2000), 377-387.

[54] H.Y. Zhu, W. Yuan, C.H. Ou. Justification for wavefront propagation in a tumour growth model with contact inhibition. Proc. R. Soc. Lond. A, 464 (2008), 1257-1273. 